Document downloaded from:

http://hdl.handle.net/10251/54684

This paper must be cited as:

Díaz Caballero, E.; Belenguer Martínez, A.; Esteban González, H.; Boria Esbert, VE. (2013).

Thru-reflect-line calibration for substrate integrated waveguide devices with tapered microstrip transitions. Electronics Letters. 49(2):132-133. doi:10.1049/el.2012.3027.

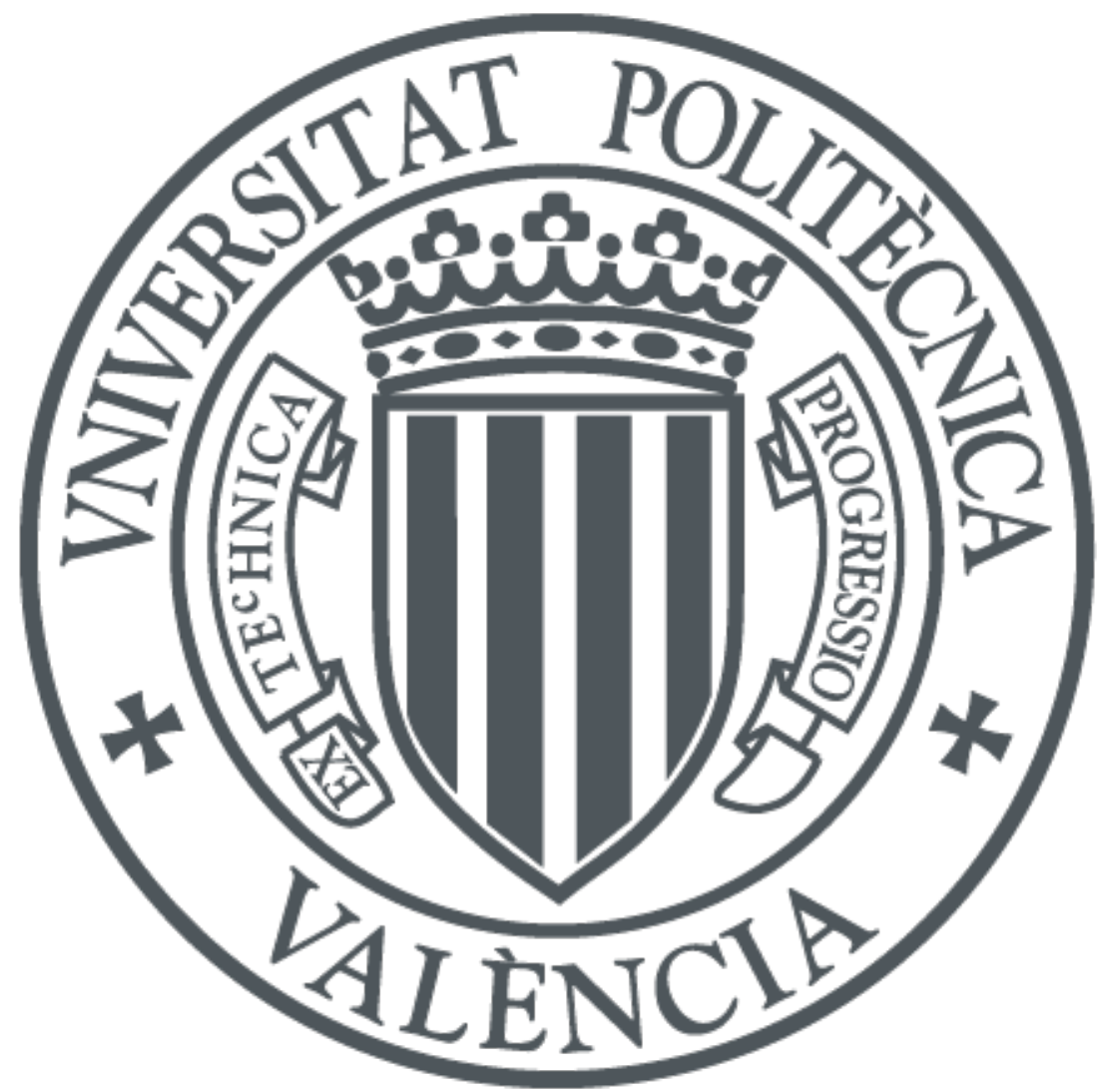

The final publication is available at

http://dx.doi.org/10.1049/el.2012.3027

Copyright Institution of Engineering and Technology (IET)

Additional Information 


\section{Thru-Reflect-Line Calibration for Substrate Integrated Waveguide Devices with Tapered Microstrip Transitions}

\author{
E. Diaz Caballero, A. Belenguer, H. Esteban and V.E. \\ Boria
}

One of the main problems when exciting or measuring substrate integrated waveguide (SIW) devices lies in the need of a good interconnection with planar structures. In this work, the negative effects produced by the connectors and the tapered microstripto-SIW transitions are de-embedded from the measurements of the SIW structure by a thru-reflect-line (TRL) calibration with an adequate and cheap SIW calibration kit.

Introduction: The interest on the relatively new type of transmission line called Substrate Integrated Waveguide (SIW) [1] is constantly increasing. This low cost realization of the traditional waveguide circuit inherits the merits from both the microstrip for easy integration and the waveguide for low radiation loss. Furthermore, it is possible to use this new technology for making many devices such as antennas, filters, multiplexers, etc $[2,3]$. However, these components must be interconnected with planar structures in order to be measured or connected to active circuits.

The tapered microstrip transition [1] has been largely adopted due to several reasons: the microstrip line is widely used; the transition covers the complete bandwidth of the SIW and the performances are better when compared to other microstrip transitions [4] or coplanar transitions [5].

However, the design of the tapered transitions is a very critical step in order to get good electromagnetic match between the microstrip line and the SIW, and although much effort is done so that the design of this transition becomes easier and faster [6], it still entails most of the mismatch loss, together with the SMA connectors and their solders.

In this work we have developed a TRL calibration kit so that all those negative effects that are masking the real response of the SIW device are removed from the measurements.

TRL calibration: First proposed by Engen and Hoer [7], the TRL calibration is usually employed in the correction of the deviations appeared in the measurements done by the network analyzer. Those deviations are due to non-idealities in the analyzer itself and the cables used to connect the device under measure to the analyzer. In the automatic network analyzers (ANA), this technique is automatically implemented so that those deviations are recognized, evaluated and, in theory, eliminated from the final measurement result. Recently, some efforts have been done in order to develop TRL calibration techniques for devices excited by planar transmission lines, such as microstrips or coplanar waveguides $[8,9]$.

Here we intend to give guidelines in order to apply the TRL approach for de-embedding the S-parameters of the SIW device also from all the effects caused by SMA connectors, tapered microstrip-to-SIW transitions and anything masking the real response of the SIW device itself.

This way, any designer or researcher can fabricate his own calibration kit adapted to this kind of technology obtaining a very good accuracy in the measurements -as you can place the reference plane right at the beginning of the SIW structure-. But it is also much cheaper than any standard commercial calibration kit, which, besides, would not be so accurate, as they can, at best, correct the deviations due to the measurement circuit between the network analyzer itself and the SMA connectors, but not those due to the microstrip line and the microstrip-to-SIW tapered transition.

TRL calibration kit for SIW: This particular SIW calibration kit (Fig. 1) has been made for a working frequency band from 5 to 12 $\mathrm{GHz}$ in a RO4003C substrate. We consider the transition to end in the second via after the taper, so we make the open (reflect) just ending there the circuit, the thru connecting the transitions of both ports and the line adding a section of SIW line between both ports.

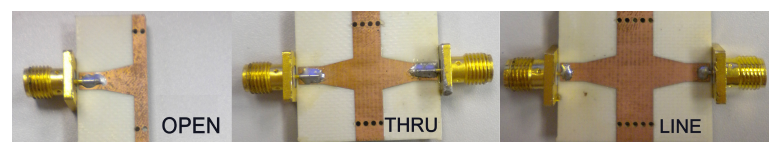

Fig. 1: TRL calibration kit for SIW devices with tapered microstrip-to-SIW transitions.

This cal kit has been designed and fabricated to correct all the systematic measurement errors, but also to de-embed SIW devices from their tapered microstrip-to-SIW transitions, as well as the microstrip line and SMA connectors. However, it is worth making it clear that this procedure could be applied to other type of transitions or excitation lines (e.g. coplanar line).

The fact that the tapered transition and the first two vias after the taper are included in all the standards of our TRL kit, places the reference plane for the measurements right in that spot, that is, at the beginning of the SIW device. That makes the measurements be quite independent from the good or bad quality of the tapered transition, as its negative effects are removed from the measurements. This can be pretty interesting when focusing in just designing or improving the SIW part of a device, as you can forget about all the negative effects of the initial transitions -which can be a whole work field, as mentioned in the introduction- and concentrate on the good performance of the SIW section.

However, in order to make a more generalized calibration kit, the reference plane could be considered to be before the tapered transition and so the TRL cal kit would have an open, a thru and a line in microstrip. This way, although the effect of the microstripto-SIW taper would not be removed, it would be a more versatile calibration kit as it could be used for any diameter and separation between via holes and for any tapered transition design.

First, we define the calibration kit in the network analyzer [10]. Then, as usual, we have to measure the whole calibration kit (Fig. 1) before measuring the SIW device so that the analyzer can correct all the systematic errors and deviations in the measure with its built-in full two-port TRL calibration function.

After calibrating, we measure the SIW device, which, in this case, is a 4-pole SIW filter (see Fig. 2). It has the same excitation microstrip line, tapered transitions and diameter and separation of the via holes than the standards of the fabricated TRL cal kit.

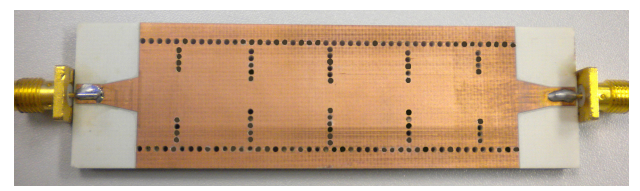

Fig. 2: SIW device to be measured and calibrated.

Results: In order to test the good performance of the presented TRL calibration, the SIW filter in Fig. 2 has been used. Figure 3 shows the comparison among its S-parameters obtained from the measurements calibrating with the $85052 \mathrm{C}$ cal kit and with the new SIW calibration kit and from the HFSS simulation of the sole 
filter (without transitions and connectors), as this is the response we would like to recover.

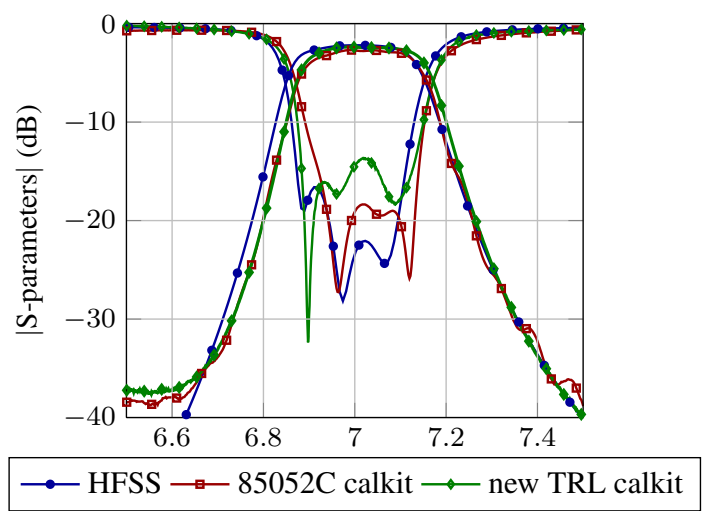

Fig. 3: Comparison among the simulated S-parameters and the measurements calibrating with the $85052 \mathrm{C}$ cal kit and with the new SIW calibration kit for the 4-pole filter in Fig. 2

It can be seen that the new SIW calibration kit corrects the frequency deviation of the poles (see Fig. 4.a), the ripple (Figs. 4.b and 4.c), the bandwidth (Fig. 4.d) and the insertion losses (Fig. 4.c) respect to the the simulated S-parameters. The frequency shift is due to the $2 \%$ tolerance in the $\varepsilon_{r}$ of the RO4003C, and the slightly worse return losses can be due to some small difference between the simulated $\tan \delta$ and the real one.

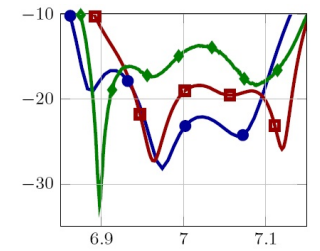

(a)

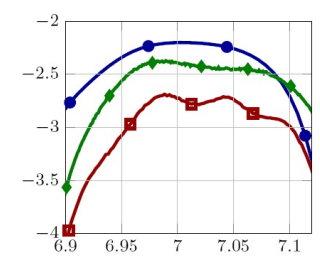

(c)

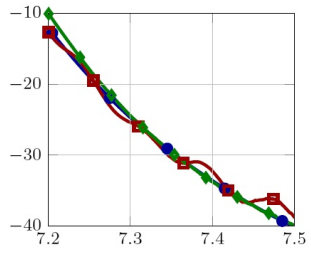

(b)

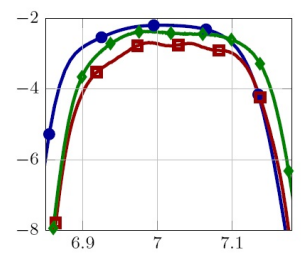

(d)
Fig. 4: Details of the improvements observed in Fig. 3 due to the new TRL cal kit for SIW.

Conclusions: A TRL calibration approach has been applied to deembed the measured S-parameters of a SIW device from all the effects of the connectors, excitation microstrip lines and tapered microstrip-to-SIW transitions -as well as all the systematic errors of the analyzer and the cables- that are masking the true response of the SIW device. This procedure is of a great interest when designers want to isolate the response of the sole SIW device, to identify how the whole structure needed for the excitation of the SIW device is modifying its response or, finally, when the designer is aware that the designed tapered transition entails a considerable mismatch loss that is masking the goodness of the designed SIW device.

This work, thus, presents a very cheap and easy way to fabricate an accurate TRL calibration kit adapted to the measurement of SIW devices.

\section{References}

1 D. Deslandes and K. Wu, "Integrated microstrip and rectangular waveguide in planar form," IEEE Microwave and Wireless Components Letters, vol. 11, no. 2, pp. 68-70, Feb. 2001.

2 M. Henry, C. Free, B. Izqueirdo, J. Batchelor, and P. Young, "Millimeter wave substrate integrated waveguide antennas: Design and fabrication analysis," Advanced Packaging, IEEE Transactions on, vol. 32, no. 1, pp. 93 -100, Feb. 2009.

3 X.-P. Chen, K. Wu, and Z.-L. Li, "Dual-band and triple-band substrate integrated waveguide filters with chebyshev and quasielliptic responses," Microwave Theory and Techniques, IEEE Transactions on, vol. 55, no. 12, pp. 2569 -2578, Dec. 2007.

4 M. Abdolhamidi, A. Enayati, M. Shahabadi, and R. FarajiDana, "Wideband single-layer dc-decoupled substrate integrated waveguide (siw) - to - microstrip transition using an interdigital configuration," in Proc. Asia-Pacific Microwave Conf. APMC 2007, 2007, pp. 1-4.

$5 \mathrm{D}$. Deslandes and $\mathrm{K}$. Wu, "Analysis and design of current probe transition from grounded coplanar to substrate integrated rectangular waveguides," IEEE Transactions on Microwave Theory and Techniques, vol. 53, no. 8, pp. 2487-2494, 2005.

6 D. Deslandes, "Design equations for tapered microstrip-toSubstrate Integrated Waveguide transitions," in Microwave Symposium Digest (MTT), 2010 IEEE MTT-S International. IEEE, 2010, pp. 704-707.

7 G. F. Engen and C. A. Hoer, "Thru-reflect-line: An improved technique for calibrating the dual six-port automatic network analyzer," vol. 27, no. 12, pp. 987-993, 1979.

8 C.-J. Chen and T.-H. Chu, "Measurement of noncoaxial multiport devices up to the intrinsic ports," Microwave Theory and Techniques, IEEE Transactions on, vol. 57, no. 5, pp. 1230 -1236 , may 2009.

$9-$, "Analytical characterization of s-matrix reconstruction transforms on multiport networks," in Microwave Conference Proceedings (APMC), 2011 Asia-Pacific, dec. 2011, pp. 1362 -1365 .

10 "Specifying calibration standards for the agilent 8510 network analyzer," Agilent Product Note 8510-5B, Tech. Rep. 